\title{
Cosmology and fundamental physics
}

\section{Gerhard Börner}

Received: 22 June 2009 / Accepted: 2 July 2009 / Published online: 16 July 2009

(C) The Author(s) 2009. This article is published with open access at Springerlink.com

\begin{abstract}
Connections between fundamental physics and cosmology are discussed.
\end{abstract}
Keywords Cosmology $\cdot$ Particle physics

\section{Introduction and outline}

Over the past ten years the parameters of the cosmological model have been well determined by astronomical observations. Supernovae of type Ia have been identified as reliable distance indicators, allowing to measure the cosmic expansion to a precision of $10 \%$. Quite surprisingly a small deviation from the linear Hubble expansion was detected indicating that the expansion is accelerating [11,12]. Detailed studies of galaxies and clusters of galaxies have revealed the existence of dark matter. These results are in full agreement with the determination of cosmic parameters from the analysis of anisotropies in the cosmic microwave background (CMB) [8].

It turns out that a simple Friedmann-Lemaître (FL) cosmological model can accommodate all the data. The following rough picture energes: the expansion time, the inverse of the Hubble constant $H_{0}$, is about 14 billion years. The total density is about equal to the critical density, defined as

$$
\rho_{c}=\frac{3 H_{0}^{2}}{8 \pi G}
$$

where $G$ is the gravitational constant.

G. Börner $(\bowtie)$

Max-Planck-Institut für Astrophysik, Postfach 1317, 85741 Garching, Germany

e-mail: grb@mpa-garching.mpg.de 
Only a small fraction of about $5 \%$ is contributed by the baryonic matter, the "normal" matter consisting of protons, neutrons and electrons. About $21 \%$ must be attributed to dark matter, clumps of nonbaryonic particles. All this adds up to $26 \%$, leaving a deficit in the balance of 74\%, something which is distributed uniformly in space. Many call this "dark energy", not a very appropriate name, because we know since Einstein that matter and energy are essentially the same. Dark Matter, and dark energy, however, are very different (see e.g. [2,9]). Whereas the mass in a comoving volume stays constant and the energy of radiation changes proportionally to the inverse of the expansion factor $R^{-1}$, the amount of dark energy in a comoving volume increases with the volume. This stuff seems to behave like a tension of empty space, relentlessly driving the expansion. "Dark energy" is a name which points to some mysterious substance, but pragmatically speaking there is no mystery, it is just another parameter introduced in the model to provide better fits to the data.

Albert Einstein had introduced a "cosmological constant" to describe the world model favored around 1915. Most astronomers at that time were convinced that the world was nothing but a uniform, infinite, unchanging assembly of stars. The attractive force of gravity would pull all the stars together into one big clump, if it were not balanced by a kind of repulsive gravity. A suitably chosen cosmological constant can provide this balance. It fell into disrepute, of course, once the expansion of the system of galaxies was discovered by Edwin Hubble.

Now, astronomers have reinstalled this quantity by the very precise measurements of the Hubble expansion. These observations are best fitted by a cosmological model with a matter content of $26 \%$ and a cosmological constant accounting for $74 \%$ of the energy content. This contribution does not necessarily have to be just a cosmological constant, it might be some kind of field energy which might change with time, although it looked like a cosmological constant now $[2,9,15]{ }^{1}$

There are as yet no convincing theoretical explanations for the dark energy but the attempts to understand it from a fundamental point of view are vigorously pursued. Here seems to be an edge, where cosmology and fundamental theories meet.

The astronomical observations are supported and confirmed by the analysis of the anisotropies of the CMB. From the power spectrum of the temperature fluctuations in the CMB sky, all the cosmic parameters can be determined [8].

This is an independent piece of evidence, and the fact that what is measured from the CMB agrees with the observations made from stars and galaxies, indicates that the universe around us is well described by a simple FL model with a precisely determined set of parameters.

But there is the need to understand the strange composition of the cosmic stuff, the embarrassing fact that the baryonic matter known to us makes up only $5 \%$ of the total, and the intriguing question why it all adds up to the critical energy density, leading to Euclidean 3-space. It seems that fundamental theories are the best chance to find an explanation.

\footnotetext{
1 Citations quoted as LNP 721 are from the book, Seiler E, Stamatescu, I.-O. (eds.) Approaches to fundamental physics, Lecture notes in physics, vol. 721. Springer, Heidelberg, 2007).
} 
In this short essay, I want to discuss some aspects of the impact of fundamental physics on cosmology, and vice versa.

Since the early 1970s, when Jürgen Ehlers and I came to the Max-Planck-Institute for Astrophysics at about the same time, I had the opportunity to discuss such questions with him and I think the need of a deeper foundation of concepts such as inflation or dark energy still exists today.

"Diese Idee bedarf noch der mathematischen Ausgestaltung" (The mathematical framework for this idea needs to be developed more) was one of his favourite statements, still true in many cases.

\section{The inflationary cosmology}

The idea that a short phase of rapid expansion-much faster than the $\propto t^{1 / 2}$ law in the radiation dominated phase of the standard model occurs in the early universe has gained wide popularity, because it seemingly explains various features of the standard model in a natural way: the high entropy, the very nearly zero curvature, or equivalently critical energy density. The spectrum of initial density fluctuations can be calculated in inflationary models and it agrees well with the measurements from the CMB. These are strong points in favour of an early stage of inflation. A scenario of this type almost appears to be necessary, because the horizon structure of the standard model without inflation would not allow a physically reasonable model of the generation of density perturbations [9].

It is an appealing scencario, but its foundations in a fundamental theory are not laid out convincingly yet. Models for inflation often employ a scalar field $\phi$ with potential energy $V(\phi)$, the "work horse" of cosmologists used also in models for spontaneous symmetry breaking, and attempts to explain dark energy ("quintessence" models).

For inflation very often a "designer" potential $V(\phi)$ is used with several local minima, and a tailor-made shape, so as to produce the desired results. A critical scrutiny reveals that those models definitely need a better "mathematical outfit".

The potential $V(\phi)$ is an effective potential, the Legendre-transform of the freeenergy. It has been shown that $V_{\text {eff }}\left(\phi_{\text {ce }}\right)$ must be convex, a property violated by the double—or more-hump designer potentials [1,10,17].

The proof of this exact result is quite straightforward: the effective potential in field theory is derived from the partition sum written as a functional of an external classical field J [1]:

$$
Z(J) \equiv\left\langle\exp \left(S\left(\phi_{c}, J\right)\right\rangle \equiv \exp (W(J))\right.
$$

where $S$ is the action.

Usually the expectation value $\phi_{c}(J)$ is defined as $\phi_{c}(J) \equiv \partial W / \partial J$, but from this the function $J\left(\phi_{c}\right)$ may not be computable.

Therefore one uses the construction

$$
\Gamma\left(\phi_{c}\right) \equiv \inf _{J}\left(W(J)-\phi_{c} J\right),
$$


and finally

$$
V_{\mathrm{eff}}\left(\phi_{c}\right)=-\lim _{V \uparrow R^{n}} \frac{1}{|V|} \Gamma\left(\phi_{v}\right)
$$

where

$$
\phi_{V}=\phi_{c}
$$

(constant) in the volume $V$.

$\mathrm{W},-\Gamma$, and $V_{\text {eff }}$ are all convex. For $W$ :

$$
\frac{\partial^{2} W}{\partial J^{2}}=-\left\langle\phi^{2}\right\rangle-\left\langle\phi_{c}\right\rangle^{2} \geq 0 .
$$

For the definition used here the proof is almost trivial. A different definition often used is the "constraint effective potential" [10], where one fixes the spatial average of $\phi$ in a volume $V$ to be $\bar{\phi}$ (by introducing a $\delta$-function in the action), and then analyses $\log Z$ with this value. The constraint effective potential need not be convex in a finite volume, but as shown in [10] it becomes convex in the thermodynamic limit, and then agrees with the standard definition.

A convex $V_{\text {eff }}$ can have straight line pieces which may not be horizontal. Each linear piece corresponds to a phase transition of first order. The action of an external field, as e.g. in a spin-system at low temperature makes the linear piece horizontal, and the coexistence of different phases is achieved. This type of inflationary models has been constructed following the ideas of "spontaneous symmetry breaking" in the Higgs model used in the standard model of particle physics [4]. In perturbation schemes the double-hump potential far the Higgs field is an adequate phenomenological description. The exact way in which the Higgs field introduces masses for the particles is probably much more involved [5].

This exact result does not apply to models of chaotic inflation, where the inflaton field starts from a high value with a high effective potential and slowly rolls down to zero. It remains to be shown, whether such freely chosen scalar fields can find a well-defined place ia fundamental theory.

It is an uncomfortable thought that inflation models appeal to the energy of a scalar field to drive the expansion of the universe, while it is quite clear (see below) that the vacuum energies of quantum field theories must not have any gravitational effect. An idea which makes this distinction plausible is certainly very desirable.

It should be kept in mind that the assumption of a classical background spacetime on which the quantum fields act is an approximation which may often not be a consistent description. Well-known cases like thermal radiation from a black hole, or particle production in an expanding universe, may be justified as long as the effects can be treated as small perturbations. But the backreaction on the classical spacetime cannot be estimated reliably. Even for the famous Hawking radiation the exact way of disappearance of the black hole is unknown. Such problems can be consistently treated only in a quantum theory of gravity. We need to wait for some time, I expect. 
A further problem arises, because in Einstein's GR different coordinate frames are equivalent, whereas canonical quantization of a field theory in different coordinate frames may lead to inequivalent quantum field theories. The predictions of such frame-dependent descriptions should be viewed with caution (radiation seen by accelerated observers in flat space-time, etc.).

Why not just insert the expectation value of the energy momentum tensor in a thermal state of the quantum field in the right hand side of Einstein's equations? If this is done for a scalar field for example, we find that this expectation value is infinite and must be regularized. It is by no means clear that for a thermal state the energy momentum tensor then is proportional to the metric

$$
\left\langle T_{\mu \nu}\right\rangle=\Lambda g_{\mu v},
$$

as it is assumed in some inflationary models.

\section{Attempts to understand dark energy}

It is fair to say that as of this time (June 2009) there does not exist an explanation of Dark Energy from fundamental physics. A nasty-minded person might add that many "models" are just a reformulation of the observational facts in the language of field theory. The popular "quintessence" model [19] makes use again of a scalar field with a tailor-made potential. The remarks on effective potentials made in the last section apply here in a similar way.

The idea of quintessence is that the cosmological constant is small, because the universe is old. One considers a uniform scalar field with potential $V(\phi)$, and postulates a value of $\phi$, such that $d V / d \phi=0$. The scalar field $\phi$ should approach this value, stay close to it and change only slowly with time. The densities of matter and energy are steadily decreasing as the cosmos evolves, and eventually the potential energy $V(\phi)$ dominates, and the universe starts an exponential expansion with the expansion factor $a \sim \exp (H t)$, and

$$
H^{2}(t) \simeq \frac{8 \pi G}{3} V(\phi)
$$

The problem is, of course, why $V(\phi)$ should be small at the value of $\phi$ for which $d V / d \phi=0$. It can, however, be set up that way, if the potential is chosen appropriately.

One can construct "tracker" solutions, where the cross-over from an early matter dominated expansion to a $\phi$-field dominated expansion can be arranged such that the cross-over occurs just after recombination, and the desired properties arise.

The task remains to imbed such a scalar field construction into a fundamental theory of particle physics. Scalar fields with the desirable effective potential just may not exist in such theories. Then again, they may be there-it is a matter of belief, but it seems a long way before it is tied up with fundamental physics.

There also is the interesting idea that the backreaction of density inhomogeneities on the expansion may produce the measured effect of an accelerated cosmic expansion [3]. 
The homogeneous FL-cosmological model is thought to result from an averaging process applied to the underlying "realistic" inhomogeneous model which describes the galaxies and their clustering. Spatial averaging in GR leads to quantities which depend on the domain over which the average is computed, and the averaged field equations differ from the field equations for the averaged quantities.

Thus the averaged FL equations contain an additional term which represents the "backreaction" of the inhomogeneities on the averaged density and pressure. The averaged scale factor $a_{D}(t)$ (averaged over a spatial domain D) obeys the generalized expnasion law

$$
3 \ddot{a}_{D}+4 \pi G a_{D}\langle\varrho\rangle_{D}-\Lambda=Q_{D}
$$

The backreaction term $Q_{D}$ depends on the inhomogeneities of the matter velocity field inside $\mathrm{D}$, and is in general non-zero.

If $Q_{D}=0$, one recovers the usual, homogeneous FL equation. A non-zero $Q_{D}$ resembles very strongly a cosmological constant.

Whether such a term can explain the Dark Energy depends on its size. It may be orders of magnitude to small, but foremost it must be calculated. If it worked, it would be a nice explanation of Dark Energy just as a GRT effect. I am skeptical, since density inhomogeneities on the scale of galaxies and clusters are large, but the fluctuations in the gravitational potential are small, of order 0.01. On the other hand, it is conceivable that mass-concentrations outside of our horizon are arranged such that their gravitational attraction produces an accelerating expansion of the galaxy system.

A third class of models for Dark Energy postulates deviations from GRT to accommodate the measurement of an accelerated expansion. A critical review of the rapidly growing literature in this area is [16]. Generally, the alternative gravity models, as e.g. the so-called $f(\mathrm{R})$ gravity (obtained by replacing the Einstein action R-2 $\Lambda$ by a function $f(\mathrm{R})$ of the Ricci scalar $\mathrm{R}$ ) gain additional freedom by a slight extension of GRT. To explain Dark Energy, however, they need extreme fine tuning, and they all seem to have severe problems of their own in addition.

\section{Cosmology and string theory}

The surprising discovery in 1998 that the expansion of the universe is accelerating $[11,12]$, had also a surprising impact on fundamental physics. The accelerating expansion can most easily be explained by a positive cosmological constant (or quite similarly an almost constant positive field energy), and the question of the cosmological constant was posed again to fundamental theories of particle physics.

One would like to relate the cosmological constant to the energy density of the vacuum state of a quantum field theory. Such a quantity is, however, formally infinite since it involves products of fields at the same space-time point. Therefore it has to be regularized and renormalized. In this context the energy density is rather an input for the renormalization than a prediction of the theory. It seems nevertheless reasonable to assume that the regularization is provided by the short distance behaviour of the products of field operators. Then a natural choice for the regularization is a cutoff at 
the energy scale where new physics is expected to appear (e.g. [4]). If one takes the Planck mass as the cutoff scale, one finds a value $10^{123}$ times bigger (for a $1 \mathrm{TeV}$ cutoff $2 \times 10^{59}$ times bigger) than the astronomical measurement of the cosmological constant.

This is certainly a phantastic failure of a theoretical prediction. This huge discrepancy indicates that these energy densities must not gravitationally be active. An extremely precise cancellation of these terms is required. At the moment there is no good theoretical idea which tells us how to achieve this. Furthermore it also casts some doubt on the models of inflation which make use of the energy of a scalar field: Why should the inflaton field have huge gravitational effects, leading to a rapid exponential expansion of the universe, while the vacuum energy of the usual particle theories has to be without any gravitational effect?

Clearly this problem would exist, even if the cosmological constant was zero. But in some sense zero is not like any other number, because one might hope that some symmetry principle could be found which would set the contribution of vacuum energies to the cosmological constant exactly to zero and kept it at that value.

In a supersymmetric field theory (SUSY) the energy density of the vacuum vanishes, since the fermionic and basonic contributions cancel each other. SUSY must, however, be a broken symmetry, because the real world is not supersymmetric. The scale must at least be about $1 \mathrm{TeV}$, and thus the discrepancy remains huge. An optimist might say $10^{59}$ is better than $10^{120}$, but it is still far from a solution of the problem. Almost certainly a new concept is required.

A small, but non-zero number cannot be derived from a symmetry principle and at the moment the question is how to obtain it from fundamental theory. Perhaps a theory of quantum gravity can illuminate this question.

Superstring theory has had an interesting connection with the cosmological constant. According to the story told in [13] (chapter 10) superstring theory in ten dimensions gives rise to a negative or zero cosmological constant, and for some time it seemed, as if the astronomical observations put a fundamental theory in trouble. Then it was discovered in the attempts to solve the "moduli problem", i.e. roughly speaking the problem of how to make the higher extra dimensions stable, that the set of sixdimensional Calabi-Yau spaces occurring in the compactification of the theory to a four-dimensional manifold had a much richer structure than anticipated.

It could be shown that in more complicated topologies the cosmological constant could acquire a small positive value [7]. This was a great relief, but there was also a strange consequence to swallow: the number of independent classical background configurations found in this approach increased tremendously. Now, one estimates that at least $\sim 10^{500}$ different solutions exist, each one giving rise to a different universe. Originally one had hoped that a unique theory of strings could be formulated, but now this hope seems to have faded away.

Would you call this a "reductio and absurdum" and give up on string theory? Well, not if you believe in string theory!

The astonishing number of $10^{500}$ string theories has led famous physicists to proclaim "a landscape of superstring vacua and a landscape of different universes [14]. The only hope to understand the properties of our universe is the appeal to an "anthropic principle", i.e. to the (trivial?) fact that we, perhaps generally intelligent life, can only 
exist in a world with specific properties [18]. This argument is merely a logical consistency, but it cannot be regarded as a physical principle. How do we know that all these other worlds really exist, and if so would not there be many other worlds which could support totally different forms of intelligent life as well?

I think it is too early for physicists to throw the towel or to "declare insolvence"- as is popular now - and hide the need for further explanation of the physics behind the anthropic principle. Surely it is quite improbable that now, in 2009, we know all about physics. It might be discovered that none of the $10^{500}$ string theories can describe a real world. Maybe they are all unstable [6] and then we would be back at the pre-string theory status without a fundamental theory. There is a chance that this may be the case. Then it would not be the "end of physics", but a new beginning.

\section{Outlook}

It is remarkable that the standard model of cosmology derived from the classical theory of GR, and from the careful study of astronomical phenomena has such deep connections to fundamental physics. In this essay I have focussed attention on some intriguing questions, but one should not forget the progress made in our understanding of the structure of the cosmos, as e.g. the possibility to explain the generation of density fluctuations with the correct spectrum and amplitude in the context of inflationary models. We should also appreciate how some basic problems, such as the role of vacuum energy, have been sharpened by viewing them in a cosmological perspective. And it is certainly quite clear that the physics of the early universe can only be studied using the tools of fundamental physics.

Seeing that there are many problems still unsolved should not give rise to pessimism. Rather an optimistic outlook is appropriate: there is a lot to discover, and the chances are great that we will understand more and more things about our world. It may well be true, as Jürgen Ehlers once remarked that "there will always be something which we have to accept unexplained" ("etwas müssen wir stets als unerklärt hinnehmen"), but that border is not yet in sight.

Acknowledgments I want to thank Erhard Seiler and Reinhard Breuer for helpful comments.

Open Access This article is distributed under the terms of the Creative Commons Attribution Noncommercial License which permits any noncommercial use, distribution, and reproduction in any medium, provided the original author(s) and source are credited.

\section{References}

1. Börner, G., Seiler, E.: Adv. Space Res. 3, 441 (1994)

2. Börner, G.: The Early Universe, 4th edn. Springer, Heidelberg (2003)

3. Buchert, T.: Gen. Relativ. Grav. 40, 467 (2008)

4. Dosch, H.G.: in LNP 127, p. 21 (2007)

5. Fredenhagen, K., Rehren, K.-U., Seiler, E.: in LNP 127, p. 61 (2007)

6. Hertog, T., Horowitz, G.T., Maeda, K.: J. High En. Phys. 0305, 60 (2003)

7. Kachru, S., et al.: hep-th/0301240 (2003)

8. Komatsu, E., et al.: Astrophys. JS 180, 330 (2009) 
9. Mukhanov, V.: Physical Foundations of Cosmology. Cambridge University Press, Cambridge (2005)

10. O’Raifeartaigh, L., Wipf, A., Yoneyama, H.: Nucl. Phys. B 271, 653 (1986)

11. Perlmutter, S., et al.: Nature 399, 551 (1998)

12. Riess, A.G., et al.: Astron. J. 116, 1009 (1998)

13. Smolin, L.: The Trouble with Physics. Houghton Mifflin Comp., New York/Boston (2006)

14. Susskind, L.: hep-th/0302219 (2003)

15. Straumann, N.: LNP 721, 327 (2007)

16. Straumann, N.: arXiv 08095148 [gr-qc] (invited talk at conf. Beyond Einstein, Mainz 2008) (2008)

17. Symanzik, K.: Commun. Math. Phys. 16, 48 (1970)

18. Weinberg, S.: hep-th/0511037 (2005)

19. Wetterich, C.: Nucl. Phys. B 252, 309 (1985) 